PDES, SUBMANIFOLDS AND

AFFINE DIFFERENTIAL GEOMETRY

BANACH CENTER PUBLICATIONS, VOLUME 69

INSTITUTE OF MATHEMATICS

POLISH ACADEMY OF SCIENCES

WARSZAWA 2005

\title{
INVARIANCE GROUPS OF RELATIVE NORMALS
}

\author{
THOMAS BINDER \\ Institute for Neuro- and Bioinformatics, University of Lübeck \\ Ratzeburger Allee 160, D-23538 Lübeck, Germany \\ E-mail:binder@inb.uni-luebeck.de \\ MARTIN WIEHE \\ Institute for Mathematics, Technische Universität Berlin \\ Strasse des 17. Juni 136, D-10623 Berlin, Germany \\ E-mail:wiehe@math.tu-berlin.de
}

\begin{abstract}
We investigate a two-parameter family of relative normals that contains Manhart's oneparameter family and the centroaffine normal. The invariance group of each of these normals is classified, and variational problems are studied. The results are Euler-Lagrange equations for the hypersurfaces that are critical with respect to the area functionals of the induced and semi-Riemannian volume forms and a classification of the critical hyperovaloids in the two-parameter family.
\end{abstract}

1. Introduction. F. Manhart [4] introduced a one-parameter family of relative normals that contains the Euclidean and the Blaschke normal. Obviously, for any two given conormals on a non-degenerate hypersurface $\mathfrak{x}$, there is a one-parameter family connecting them. This family is unique up to affine reparametrizations.

For example, suppose $\mathfrak{x}$ is a non-degenerate centroaffine hypersurface immersion, then the Euclidean support function $\rho^{E}$ never vanishes. One can add another parameter to Manhart's family which joins the centroaffine normal, i.e.

$$
\mathfrak{Y}^{(a, b)}=\left(\rho^{E}\right)^{-b}\left|H_{n}^{E}\right|^{-a} \mu, \quad a, b \in \mathbb{R} .
$$

Here $\mu, \rho^{E}, H_{n}^{E}$ denote the Euclidean conormal, support function, and Gauss-Kronecker curvature, respectively. (The sign is fixed by $\rho^{E}>0$.)

For a relative normal $\mathfrak{y}$ for $\mathfrak{x}$ we define the area functionals with respect to the induced and semi-Riemannian volume forms $\omega$ and $\hat{\omega}$ by

$$
A=A(M, \mathfrak{x}, \mathfrak{y}):=\int_{M} \omega, \quad \text { and } \quad \hat{A}=\hat{A}(M, \mathfrak{x}, \mathfrak{y}):=\int_{M} \hat{\omega} .
$$

Let $A^{(a, b)}:=A\left(M, \mathfrak{x}, \mathfrak{y}^{(a, b)}\right)$ and $\hat{A}^{(a, b)}:=\hat{A}\left(M, \mathfrak{x}, \mathfrak{y}^{(a, b)}\right)$.

2000 Mathematics Subject Classification: Primary 53A15; Secondary 53A10.

The paper is in final form and no version of it will be published elsewhere. 
In affine differential geometry the Blaschke area $A^{e}:=A^{\left(\frac{1}{4}, 0\right)}$ is one of the best analysed functionals. Work in this direction was started by Blaschke [1] for dimension two. The first and second variation of $A^{e}$ for arbitrary dimension had been studied by Calabi [2]; further contributions include [5] and [10]. Another approach is to use $\hat{A}$, which was followed by [3], [4]. Wang [11] studies the first and second variation of the centroaffine area $\hat{A}^{c}:=\hat{A}^{(0,1)}$.

The first and second variation of $A^{(a, 0)}$ in Manhart's one-parameter family were studied by the second author in [12]. Results for the first variation of $A^{(0, b)}$ can be found in [7]. In this paper we derive Euler-Lagrange equations for the first variation of $A^{(a, b)}$ and $\hat{A}^{(a, b)}$ and prove

TheOREM 1. Let $\mathfrak{x}: M^{n} \rightarrow A^{n+1}$ be a hyperovaloid which is $A^{(a, b)}$-critical and suppose $(a, b) \neq(1,0)$. Then $\mathfrak{x}(M)$ is a sphere.

REMARK 2. Any hypersurface is $A^{(1,0)}$-critical.

2. Relative geometry of hypersurfaces. For a detailed introduction to the subject see e.g. [6] or [9].

Consider a non-degenerate $C^{\infty}$-immersion $\mathfrak{x}: M^{n} \rightarrow A^{n+1}$ of an $n$-dimensional, $n \geq 2$, connected oriented $C^{\infty}$-manifold into real flat affine space with standard flat connection $\bar{\nabla}$. Suppose that $\mathfrak{y}: M^{n} \rightarrow \mathbb{R}^{n+1}$ is a $C^{\infty}$ transversal vector field along $\mathfrak{x}$, i.e. $d \mathfrak{x}\left(T_{p} M\right) \oplus$ $\mathbb{R} \mathfrak{n}(p)=\mathbb{R}^{n+1}$ at each $p \in M$. The vector space associated to $A^{n+1}$ is denoted by $\mathbb{R}^{n+1}$. The structure equations of $\mathfrak{x}$ with respect to $\mathfrak{y}$ read as follows:

$$
\bar{\nabla}_{u} d \mathfrak{x}(v)=d \mathfrak{x}\left(\nabla_{u} v\right)+h(u, v) \mathfrak{y}, \quad d \mathfrak{y}(u)=-d \mathfrak{x}(S u)+\theta(u) \mathfrak{y}
$$

for all vector fields $u, v \in \mathfrak{X}(M)$. If $\mathfrak{y}$ has vanishing connection form $\theta$, then it is called a relative normal. From now on we will always assume that $\mathfrak{y}$ is a relative normal. In this case the pair $(\mathfrak{x}, \mathfrak{y})$ is called a relative hypersurface.

$h$ is a symmetric bilinear form which is also non-degenerate since $\mathfrak{x}$ is non-degenerate; it is hence called the relative metric induced by $\mathfrak{y}$. We denote the Levi-Civita connection of $h$ and the positive valued semi-Riemannian volume form of $h$ by $\hat{\nabla}$ and $\hat{\omega}$, respectively. $\nabla$ is a torsion-free Ricci-symmetric affine connection called the induced connection. $S$ is called the shape operator. Its trace $n H:=\operatorname{trace} S$ is the relative mean curvature and its determinant $H_{n}:=\operatorname{det} S$ is the relative Gauss-Kronecker curvature. The induced volume form $\omega$ is defined by

$$
\omega\left(u_{1}, \ldots, u_{n}\right):=\operatorname{det}\left(d \mathfrak{x}\left(u_{1}\right), \ldots, d \mathfrak{x}\left(u_{n}\right), \mathfrak{y}\right)
$$

it is parallel with respect to the induced connection: $\nabla \omega=0$.

Define the (1,2)-difference tensor $C$, the Tchebychev vector field $T$ and the Tchebychev form $T^{b}$ by

$$
C(u, v)=C_{u} v:=\nabla_{u} v-\hat{\nabla}_{u} v, \quad n h(T, u)=n T^{b}(u):=\operatorname{trace}\{v \mapsto C(v, u)\} .
$$

Generally, ${ }^{b}$ denotes the operation of lowering an index with respect to $h$. 
Often it will be convenient to consider the conormal $\mathfrak{Y}$ to describe the normalization of a hypersurface, which is defined as a section of the cotangent line bundle satisfying

$$
\langle\mathfrak{Y}, d \mathfrak{x}\rangle=0 \quad \text { and } \quad\langle\mathfrak{Y}, \mathfrak{y}\rangle=1
$$

where $\langle\cdot, \cdot\rangle:\left(\mathbb{R}^{n+1}\right)^{*} \times \mathbb{R}^{n+1} \rightarrow \mathbb{R}$ denotes the standard scalar product. When talking about a relative normalization, we mean that either $\mathfrak{Y}$ or $\mathfrak{y}$ is given on $\mathfrak{x}$. This makes sense, since for relative hypersurfaces there is a bijective correspondence between normals and conormals.

The relative support function with respect to a point $\mathfrak{x}_{0} \in A^{n+1}$ is defined by $\rho_{\mathfrak{x}_{0}}:=$ $\left\langle\mathfrak{Y}, \mathfrak{x}_{0}-\mathfrak{x}\right\rangle$. Let $\Delta$ denote the Laplacian with respect to $\hat{\nabla}$. We define the Laplace-type operators

$$
\square f:=\Delta f+n T^{b}\left(\operatorname{grad}_{h} f\right), \quad \square^{*} f:=\Delta f-n T^{b}\left(\operatorname{grad}_{h} f\right) .
$$

The induced quantities are invariant with respect to the full affine group $G L(n+$ $1, \mathbb{R}) \oplus \mathbb{R}^{n+1}$ acting on $\mathbb{R}^{n+1}$ in the following sense: For any given relative hypersurface $(\mathfrak{x}, \mathfrak{y})$ and $(B, \mathfrak{b}) \in G L(n+1, \mathbb{R}) \oplus \mathbb{R}^{n+1}$, the coefficients of the structure equations of $(\mathfrak{x}, \mathfrak{y})$ and $\left(\mathfrak{x}^{\natural}:=B \mathfrak{x}+\mathfrak{b}, \mathfrak{y}^{\natural}:=B \mathfrak{y}\right)$ coincide: $\nabla=\nabla^{\natural}, h=h^{\natural}$, and $S=S^{\natural}$.

We now list some formulas describing the change of relative normalization.

Lemma 1. For a hypersurface $\mathfrak{x}: M^{n} \rightarrow A^{n+1}$, any two conormals $\mathfrak{Y}$ and $\mathfrak{Y}^{\natural}$ with the same orientation are related by $\mathfrak{Y}^{\natural}=e^{\varphi} \mathfrak{Y}$, where $\varphi \in C^{\infty}(M)$. Under this transition, the relative metric changes conformally: $h^{\natural}=e^{\varphi} h$. Moreover, we compute (see e.g. [9])

$$
\begin{aligned}
\mathfrak{y}^{\natural} & =e^{-\varphi}(\mathfrak{y}+d x(\operatorname{grad} \varphi)), \\
\nabla_{u}^{\natural} v & =\nabla_{u} v-h(u, v) \operatorname{grad} \varphi, \\
\Delta^{\natural} f & =e^{-\varphi}\left(\Delta f+\frac{n-2}{2} d \varphi(\operatorname{grad} f)\right), \\
\hat{\omega}^{\natural} & =e^{\frac{n}{2} \varphi} \hat{\omega}, \\
\omega^{\natural} & =e^{-\varphi} \omega, \\
S^{\natural} u & =e^{-\varphi}\left(S u-\nabla_{u} \operatorname{grad} \varphi+u(\varphi) \operatorname{grad} \varphi\right), \\
H^{\natural} & =e^{-\varphi}\left(H-\frac{1}{n} \Delta \varphi-T^{b}(\operatorname{grad} \varphi)+\frac{1}{n}\|\operatorname{grad} \varphi\|^{2}\right), \\
T^{\natural \natural} & =T^{b}-\frac{n+2}{2 n} d \varphi, \\
T^{\natural} & =e^{-\varphi}\left(T-\frac{n+2}{2 n} \operatorname{grad} \varphi\right) .
\end{aligned}
$$

Finally, let us mention some special relative normals.

(i) The Blaschke normal $\mathfrak{y}^{e}$ is determined up to sign by $|\omega|=\hat{\omega}$, which is called the apolarity condition; it is also characterized up to a non-vanishing constant factor by $T=0$. The Blaschke normal is invariant with respect to unimodular affine transformations $S L(n+1, \mathbb{R}) \oplus \mathbb{R}^{n+1}$, meaning that for any unimodular affine transformation $(B, \mathfrak{b})$ the Blaschke normal of $\mathfrak{x}^{\natural}=B \mathfrak{x}+\mathfrak{b}$ is $\mathfrak{y}^{\natural e}=B \mathfrak{y}^{e}$. Invariants induced by the Blaschke normal will be denoted by ${ }^{e}$. 
(ii) For an appropriate choice of an origin, any non-degenerate hypersurface locally can be endowed with $\mathfrak{y}^{c}:=-\mathfrak{x}$, which is the centroaffine normal. It is characterized by $S=$ id. Therefore, a proper relative sphere is exactly the underlying hypersurface with its centroaffine normal up to a constant factor. The centroaffine normal is invariant with respect to $G L(n+1, \mathbb{R})$. Centroaffine invariants will be marked by ${ }^{c}$ if ambiguous.

(iii) Locally, we can normalize any hypersurface with a constant transversal field, which is always a relative normal. The hypersurface will be an improper relative sphere with respect to this normal.

(iv) The Euclidean normal is a relative normal which is invariant with respect to the group of Euclidean motions $S O(n+1, \mathbb{R}) \oplus \mathbb{R}^{n+1}$. Euclidean invariants will be marked by ${ }^{E}$ if ambiguous. Moreover, we denote fundamental forms by I, II $:=h^{E}$ and write $\mu:=\mathfrak{Y}^{E}=\mathfrak{y}^{E}$.

3. Invariance groups of constructions of relative normals. The construction of a relative normal is a mapping which assigns a relative normal $\mathfrak{y}$ to a given non-degenerate hypersurface $\mathfrak{x}$. The invariance group of such a construction is the maximal subgroup $I \subseteq G L(n+1, \mathbb{R}) \oplus \mathbb{R}^{n+1}$ such that the order of construction and transformation does not matter, i.e. for any $g \in I$ with linear part $B$ we have $c \circ g=B \circ c$ on the set of all non-degenerate hypersurfaces.

Examples of constructions are $E, e$ and $c$. Of course, we are only interested in a small subset of all constructions, namely those with big invariance groups. In the generic case, invariance groups will be $\{(i d, 0)\}$. The invariance groups of relative normals in the two-parameter family will be denoted by $I^{(a, b)}$.

Lemma 2. Let $\mathfrak{x}: M^{n} \rightarrow A^{n+1}$ be a non-degenerate hypersurface. For a given conormal $\mathfrak{Y}$ and $q \in C^{\infty}(M)$, let $\mathfrak{Y}^{(a)}=q^{a} \mathfrak{Y}$ be a one-parameter family of relative conormals. Assume $\rho=\rho^{(0)} \neq 0$. Let $G$ be a subgroup of the full affine group such that $G \subseteq I^{\left(a_{0}\right)}$ and $G \subseteq I^{\left(a_{1}\right)}$ for two values $a_{0} \neq a_{1}$. Then $G \subseteq I^{(a)}$ for all $a \in \mathbb{R}$.

Proof. Without loss of generality we can assume $a_{0}=0$ and $a_{1}=1$, for otherwise the oneparameter family $\mathfrak{Y}^{(a)}=\tilde{q}^{\tilde{a}} \tilde{\mathfrak{Y}}$ where $\tilde{q}=q^{a_{1}-a_{0}}, \tilde{a}=\frac{a-a_{0}}{a_{1}-a_{0}}$ and $\tilde{\mathfrak{Y}}=q^{a_{0}} \mathfrak{Y}$ satisfies this condition. The proof is trivial for a pure translation, so assume the affine map from $G$ fixes the origin and has matrix $B$. Let $\mathfrak{x}^{\natural}=B \mathfrak{x}, \mathfrak{y}^{\natural}=B \mathfrak{y}^{(a)}$ be the transformed hypersurface. It suffices to prove $\mathfrak{y}^{\natural(a)}=\mathfrak{y}^{\natural}$. We know that $\mathfrak{y}^{\natural(0)}=B \mathfrak{y}^{(0)}$ and $\mathfrak{y}^{\natural(1)}=B \mathfrak{y}^{(1)}$, thus $\mathfrak{Y}^{\natural(0)}=B^{*-1} \mathfrak{Y}^{(0)}$ and $\mathfrak{Y}^{\natural(1)}=B^{*-1} \mathfrak{Y}^{(1)}$. From the assumption we can express $q=\frac{\rho^{(1)}}{\rho^{(0)}}$. We get $\rho^{\natural(0)}=\rho^{(0)}$ and $\rho^{\natural(1)}=\rho^{(1)}$, hence $q^{\natural}=q$. Finally we get

$$
\left\langle\mathfrak{Y}^{\natural(a)}, \mathfrak{y}^{\natural}\right\rangle=\left\langle q^{\natural a} B^{*-1} \mathfrak{Y}, B q^{-a}\left(\mathfrak{y}+d \mathfrak{x} \operatorname{grad} \log q^{a}\right)\right\rangle=1 .
$$

COROLlaRY 3. (i) In the particular case that $I^{\left(a_{0}\right)} \subseteq I^{\left(a_{1}\right)}$ it follows that $I^{(a)} \cap I^{\left(a_{1}\right)}=$ $I^{\left(a_{0}\right)}$ for each $a \in \mathbb{R} \backslash\left\{a_{1}\right\}$.

(ii) If $I^{\left(a_{0}\right)} \subseteq I^{\left(a_{1}\right)}=G L(n+1, \mathbb{R}) \oplus \mathbb{R}^{n+1}$, then $I^{(a)}=I^{\left(a_{0}\right)}$ for each $a \in \mathbb{R} \backslash\left\{a_{1}\right\}$.

The following theorem is an extension of [12], Theorem 5.7. 
TheOREM 4. Let $\mathfrak{x}: M^{n} \rightarrow A^{n+1}$ be a non-degenerate hypersurface and $a, b \in \mathbb{R}$. The invariance group of the relative normal $\mathfrak{y}^{(a, b)}$ is

(i) $S L(n+1, \mathbb{R}) \oplus \mathbb{R}^{n+1}$ if $(a, b)=\left(\frac{1}{n+2}, 0\right)$,

(ii) $\mathbb{R}^{+} S O(n+1, \mathbb{R}) \oplus \mathbb{R}^{n+1}$ if $(a, b)=\left(-\frac{1}{n}, 0\right)$ (in this case $\mathfrak{y}^{(a, b)}$ is called the conformal relative normal),

(iii) $S O(n+1, \mathbb{R}) \oplus \mathbb{R}^{n+1}$ if $a \notin\left\{\frac{1}{n+2},-\frac{1}{n}\right\}, b=0$,

(iv) $G L(n+1, \mathbb{R})$ if $(a, b)=(0,1)$,

(v) $S L(n+1, \mathbb{R})$ if $a(n+2)+b=1$ and $y^{(a, b)}$ is neither the Blaschke nor the centroaffine normal,

(vi) $\mathbb{R}^{+} S O(n+1, \mathbb{R})$ if $b=$ an +1 and $y^{(a, b)}$ is neither the conformal nor the centroaffine normal,

(vii) $S O(n+1, \mathbb{R})$ otherwise.

Proof. As $\rho^{E}$ is not invariant with respect to translations (origin is fixed), we know that translations are not part of the invariance group for $b \neq 0$. We exclude the line $a(n+2)+b=1$. This part of the proof follows easily from Lemma 2 , where one has to use invariance groups of the centroaffine and the Blaschke normalizations.

Invariance. Suppose we have a linear transformation $\mathfrak{x}^{\natural}=B \mathfrak{x}$ of $\mathfrak{x}$ such that $B=c D$ for some $D \in O(n+1, \mathbb{R})$ and $0 \neq c \in \mathbb{R}$. Define $\mathfrak{y}^{\natural}=B \mathfrak{y}^{(a, b)}$. It is our aim to find conditions under which $\mathfrak{y}^{\natural}=\mathfrak{y}^{(a, b) \natural}$, where $\mathfrak{y}^{(a, b)}$ is the relative normal of $\mathfrak{x}^{\natural}$ belonging to the parameter $(a, b)$. We have $d \mathfrak{x}^{\natural}=c D d \mathfrak{x}$ and thus $\mu^{\natural}=D \mu$. The sign of $\mu^{\natural}$ is chosen such that

$$
\rho^{E \natural}=-\left\langle\mu^{\natural}, \mathfrak{x}^{\natural}\right\rangle=-\langle D \mu, c D \mathfrak{x}\rangle=-c\langle\mu, \mathfrak{x}\rangle=c \rho^{E} .
$$

Moreover,

$$
-d \mathfrak{x}^{\natural}\left(S^{E \natural} u\right)=d \mu^{\natural}(u)=D d \mu(u)=-\epsilon D d \mathfrak{x}\left(S^{E} u\right)=-c^{-1} d \mathfrak{x}^{\natural}\left(S^{E} u\right) .
$$

We get $S^{\natural E}=c^{-1} S^{E}$, hence $H_{n}^{\natural E}=c^{-n} H_{n}^{E}$. Finally,

$$
\mathfrak{Y}^{(a, b) \natural}=\rho^{E \natural-b}\left|H_{n}^{E \natural}\right|^{-a} \mu^{\natural}=c^{a n-b} D \mathfrak{Y}^{(a, b)}
$$

and

$$
\left\langle\mathfrak{Y}^{(a, b) \natural}, \mathfrak{y}^{\natural}\right\rangle=c^{a n-b}\left\langle D \mathfrak{Y}^{(a, b)}, B \mathfrak{y}^{(a, b)}\right\rangle=c^{a n-b+1}\left\langle D \mathfrak{Y}^{(a, b)}, D \mathfrak{y}^{(a, b)}\right\rangle=c^{a n-b+1}=1 .
$$

This works only for $c=1$ or $b=a n+1$.

Maximality. Suppose that $\mathfrak{x}, \mathfrak{x}^{\natural}: M^{n} \rightarrow A^{n+1}$ are non-degenerate hypersurfaces such that $\mathfrak{x}^{\natural}=B \mathfrak{x}$ and $\mathfrak{y}^{(a, b) \natural}=B \mathfrak{y}^{(a, b)}$, where $B \in G L(n+1, \mathbb{R})$. Then all objects induced on $M$ by $\left(\mathfrak{x}, \mathfrak{y}^{(a, b)}\right)$ and $\left(\mathfrak{x}^{\natural}, \mathfrak{y}^{(a, b) \natural}\right)$ coincide. Let us show that we can write $B=c D$ for some $D \in S O(n+1, \mathbb{R})$ and $c \in \mathbb{R} \backslash\{0\}$, where $c=1$ follows from the invariance part. This is done if we prove $\nabla\left(\mathrm{I}^{\natural}\right)=\nabla(\mathrm{I})$ and $\mathbb{I}^{\natural}=c \mathbb{I}$. The definition can be rewritten as

$$
\mathfrak{Y}^{(a, b)}=\rho^{E-a(n+2)-b} \rho^{e a(n+2)} \mu=\rho^{E-a(n+2)-b+1} \rho^{e a(n+2)-1} \mathfrak{Y}^{e} .
$$

We have

$$
T^{b(a, b)}=\frac{n+2}{2 n} d \log \left(\rho^{E a(n+2)+b-1} \rho^{e 1-a(n+2)}\right)
$$


With $T^{b(a, b)}=T^{b(a, b)}$ घ we get

$$
\left(\frac{\rho^{e \natural}}{\rho^{e}}\right)^{1-a(n+2)}\left(\frac{\rho^{E \natural}}{\rho^{E}}\right)^{a(n+2)+b-1}=\text { const } \neq 0 .
$$

Under a $G L(n+1, \mathbb{R})$ transformation of the hypersurface the Blaschke support function is changed by a constant factor which equals the determinant of the transformation matrix. Thus, for $a(n+2)+b \neq 1$, we get that $\rho^{\natural E} / \rho^{E}=$ const. We obtain $\mathbb{I}^{\natural}=c \mathbb{I}$ from

$$
\rho^{E-a(n+2)-b} \rho^{e a(n+2)} \mathbb{I}=h^{(a, b)}=h^{\natural(a, b)}=\rho^{E \natural-a(n+2)-b} \rho^{e \natural a(n+2)} \mathbb{I}^{\natural} .
$$

The proof is finished by recalling $\nabla^{\natural(a, b)}=\nabla^{(a, b)}$ in

$$
\begin{aligned}
\nabla_{u}^{(a, b){ }_{u}^{\natural}} v & =\nabla\left(\mathrm{I}^{\natural}\right)_{u} v-\mathbb{I}^{\natural}(u, v) \operatorname{grad}\left(\mathbb{I}^{\natural}\right) \log \left(\rho^{\natural E-a(n+2)-b} \rho^{e \natural a(n+2)}\right) \\
& =\nabla\left(\mathrm{I}^{\natural}\right)_{u} v-\mathbb{I}(u, v) \operatorname{grad}(\mathbb{I}) \log \left(\rho^{E-a(n+2)-b} \rho^{e a(n+2)}\right) \\
& =\nabla\left(\mathrm{I}^{\natural}\right)_{u} v+\nabla_{u}^{(a, b)} v-\nabla(\mathrm{I})_{u} v .
\end{aligned}
$$

The classification follows from the unification of the two parts.

We conclude this section by mentioning another possibility of a one-parameter family. TheOREm 5. Suppose $\operatorname{det} S^{e} \neq 0$. Then $\mathfrak{Y}^{(a)}=\left|H_{n}{ }^{e}\right|^{-a} \mathfrak{Y}^{e}$ is a one-parameter family with invariance group

(i) $G L(n+1, \mathbb{R}) \oplus \mathbb{R}^{n+1}$ if $a=-\frac{2(n+1)}{n^{2}}$, and

(ii) $S L(n+1, \mathbb{R}) \oplus \mathbb{R}^{n+1}$ otherwise.

Proof. First observe that translations are included in the invariance groups since the construction is translation independent. For $B=c D \in G L(n+1, \mathbb{R})$ where $c>0$ and $\operatorname{det} D= \pm 1$ suppose $\mathfrak{x}^{\natural}=B \mathfrak{x}$ and let $\mathfrak{y}^{\natural}=B \mathfrak{y}^{(a)}$. As in the previous proof, we ask for $\mathfrak{y}^{\natural}=\mathfrak{y}^{(a) \natural}$. As before we get $S^{e \natural}=c^{-\frac{n}{n+2}} S^{e}$, thus $H_{n}{ }^{e \natural}=c^{-\frac{n^{2}}{n+2}} H_{n}{ }^{e}$. We obtain $\mathfrak{Y}^{(a) \natural}=c^{\frac{n(1+a n)}{n+2}} D^{*-1} \mathfrak{Y}^{(a)}$. Finally, $\left\langle\mathfrak{Y}^{(a)}, \mathfrak{y}^{\natural}\right\rangle=c^{\frac{a n^{2}+2 n+2}{n+2}}$ shows that either $c=1$ or $a=-\frac{2(n+1)}{n^{2}}$. This shows that the normals are invariant with respect to the given groups. The maximality follows from Corollary 3 (ii).

For hypersurfaces with non-singular Blaschke shape operator, there is a relative normalization which is invariant with respect to the full affine group.

Corollary 6. Consider a one-parameter family $\mathfrak{Y}^{(a)}=\left|H_{n}\right|^{-a} \mathfrak{Y}$ with $H_{n} \neq 0$. Then $I^{(0)} \subseteq I^{(a)}$ for all $a \in \mathbb{R}$.

4. First variation of area functionals. To do variational calculus we follow the notation of [12]. A relative deformation of a hypersurface $\mathfrak{x}$ with relative normal $\mathfrak{y}$ is a $C^{\infty}$-family $\left(\mathfrak{x}^{t}, \mathfrak{y}^{t}\right)$ of non-degenerate relative hypersurfaces such that $\mathfrak{x}^{0}=\mathfrak{x}$ and $\mathfrak{y}^{0}=\mathfrak{y}$. We describe an infinitesimal deformation of $\mathfrak{x}$ by the pair $(\psi, \phi)$ defined by

$$
\mathfrak{x}^{\prime}=d \mathfrak{x}(\psi)+\phi \mathfrak{y}, \quad(.)^{\prime}:=\left.\frac{\partial}{\partial t}(.)\right|_{t=0}
$$

We will use the following formulas from [12], Lemma 3.4 and (6.1.3), which hold for any relative deformation.

$$
\left\langle\mathfrak{Y}^{\prime}, d \mathfrak{x}\right\rangle=-\psi^{b}-d \phi,
$$




$$
\begin{aligned}
(\log \omega)^{\prime} & =\left\langle\mathfrak{Y}, \mathfrak{y}^{\prime}\right\rangle-n H \phi+\operatorname{div} \psi, \\
(\log \hat{\omega})^{\prime} & =\frac{1}{2}\left(-n\left\langle\mathfrak{Y}, \mathfrak{y}^{\prime}\right\rangle+\square^{*} \phi-n H \phi+2 \operatorname{div} \psi\right), \\
\rho^{\prime} & =-\rho\left\langle\mathfrak{Y}, \mathfrak{y}^{\prime}\right\rangle+h(\operatorname{grad} \rho, \psi+\operatorname{grad} \phi)-\phi .
\end{aligned}
$$

Moreover, for a Euclidean deformation (i.e. $\mathfrak{y}^{t}$ is the Euclidean normal of $\mathfrak{x}^{t}$ ) with infinitesimal representation $(\tilde{\psi}, \tilde{\phi})$ we obtain (cf. [12], (4.1.2b))

$$
\left(\log H_{n}^{E}\right)^{\prime}=\square^{E} \tilde{\phi}+n H^{E} \tilde{\phi}-2 n T^{b E}(\tilde{\psi}+\operatorname{grad}(\mathbb{I}) \tilde{\phi}) .
$$

Proposition 7. Let $\mathfrak{x}: M^{n} \rightarrow A^{n+1}$ be a non-degenerate hypersurface and $a, b \in \mathbb{R}$. Then

(i) $\mathfrak{x}$ is $A^{(a, b)}$-critical if and only if

$$
(a-1) n H^{(a, b)}-\frac{b}{\rho^{(a, b)}}=0 .
$$

(ii) $\mathfrak{x}$ is $\hat{A}^{(a, b)}$-critical if and only if

$$
(1+a n)\left(\operatorname{div}\left(\hat{\nabla}^{(a, b)} T^{(a, b)}\right)-H^{(a, b)}\right)+\frac{b}{\rho^{(a, b)}}=0 .
$$

Proof. Fix $(a, b)$ and assume that for each deformed hypersurface $\mathfrak{x}^{t}$ the deformed normal is $\mathfrak{y}^{(a, b) t}$ from the two-parameter family. Then

$$
d \mathfrak{x}(\psi)+\phi \mathfrak{y}^{(a, b)}=\mathfrak{x}^{\prime}=d \mathfrak{x}(\tilde{\psi})+\tilde{\phi} \mu
$$

links the two representations. We will first compute the unknown part $\left\langle\mathfrak{Y}^{(a, b)}, \mathfrak{y}^{(a, b) \prime}\right\rangle$ in the formulas above.

$$
\begin{aligned}
\left\langle\mathfrak{Y}^{(a, b)}, \mathfrak{y}^{(a, b) \prime}\right\rangle= & -\left\langle\left(\rho^{E-b}\left|H_{n}^{E}\right|^{-a} \mu\right)^{\prime}, \rho^{E b}\left|H_{n}^{E}\right|^{a}\left(\mu+d \mathfrak{x}\left(\operatorname{grad} \log \left(\rho^{E-b}\left|H_{n}^{E}\right|^{-a}\right)\right)\right)\right\rangle \\
= & a H_{n}^{E-1}\left(H_{n}^{E \prime}-\mathbb{I}\left(\operatorname{grad}(\mathbb{I}) H_{n}^{E}, \tilde{\psi}+\operatorname{grad}(\mathbb{I}) \tilde{\phi}\right)\right) \\
& +b \rho^{E-1}\left(\rho^{E \prime}-\mathbb{I}\left(\operatorname{grad}(\mathbb{I}) \rho^{E}, \tilde{\psi}+\operatorname{grad}(\mathbb{I}) \tilde{\phi}\right)\right) \\
= & a\left(\square^{(a, b)} \phi+n H^{(a, b)} \phi\right)-b \rho^{(a, b)-1} \phi .
\end{aligned}
$$

We used the fact $\tilde{\phi}=\rho^{E b}\left|H_{n}^{E}\right|^{a} \phi$. For the first part of the assertion we compute, using (3) and (7),

$$
\begin{aligned}
\left(A^{(a, b)}\right)^{\prime} & =\int \omega^{(a, b) \prime}=\int\left(\left\langle\mathfrak{Y}^{(a, b)}, \mathfrak{y}^{(a, b) \prime}\right\rangle-n H^{(a, b)} \phi+\operatorname{div}\left(\nabla^{(a, b)}\right) \psi\right) \omega^{(a, b)} \\
& =\int\left(a \square^{(a, b)} \phi+(a-1) n H^{(a, b)} \phi-b \rho^{(a, b)-1} \phi\right) \omega^{(a, b)} .
\end{aligned}
$$

Now (i) follows by the fundamental theorem since $\int \square^{(a, b)}(\cdot) \omega^{(a, b)}=0$. For the second part (ii),

$$
\begin{aligned}
\left(\hat{A}^{(a, b)}\right)^{\prime} & =\int \hat{\omega}^{(a, b) \prime}=\frac{1}{2} \int\left(-n\left\langle\mathfrak{Y}^{(a, b)}, \mathfrak{y}^{(a, b) \prime}\right\rangle+\square^{*(a, b)} \phi-n H^{(a, b)} \phi\right) \hat{\omega}^{(a, b)} \\
& =\frac{1}{2} \int\left(\left(\square^{*(a, b)}-a n \square^{(a, b)}\right) \phi-(1+a n) n H^{(a, b)} \phi+n b \rho^{(a, b)-1} \phi\right) \hat{\omega}^{(a, b)} \\
& =\frac{n}{2} \int\left((1+a n) \operatorname{div}{ }^{(a, b)} T^{(a, b)}-(1+a n) H^{(a, b)}+b \rho^{(a, b)-1}\right) \phi \hat{\omega}^{(a, b)}
\end{aligned}
$$


where we have used (4), (7), the definitions of $\square, \square^{*}$ and the identity

$$
\left.\int T^{b}(\operatorname{grad} \phi) \omega=-\int h\left(\operatorname{grad} \phi, \operatorname{grad} \log \left|\frac{\omega}{\hat{\omega}}\right|\right)\right) \omega=\int(\operatorname{div} T) \phi \omega .
$$

We will now prove Theorem 1. Observe that by applying the first Minkowski integral formula

$$
0=\int\left(1-\rho^{(a, b)} H^{(a, b)}\right) \omega^{(a, b)}=\frac{n(a-1)-b}{n(a-1)} \int \omega^{(a, b)}
$$

we get $b=n(a-1)$. Proposition 7 (i) states that there is the relation $H=f(\rho)=\frac{1}{\rho}$ for some function $f$ on the real line. The assertion follows from the following theorem of U. Simon for the first relative curvature function, which is the mean curvature.

TheOREM 8 ([8], Theorem 6.1). Let $\mathfrak{x}: M^{n} \rightarrow A^{n+1}$ be a closed locally strongly convex $C^{5}$-hypersurface with a relative normal $\mathfrak{y}$. Suppose $H>0$. Assume that there exists a $C^{1}$-function $f$ such that $H=f(\rho)$ and $f^{\prime} \leq 0$. Then $\mathfrak{x}(M)$ is a sphere.

\section{References}

[1] W. Blaschke, Vorlesungen über Differentialgeometrie, vol. II, Springer, Berlin, 1923.

[2] E. Calabi, Hypersurfaces with maximal affinely invariant area, Am. J. Math. 104 (1982), 91-126.

[3] E. Glässner, Über die Minimalflächen der zweiten Fundamentalform, Monatsh. Math. 78 (1974), 193-213.

[4] F. Manhart, Zur Differentialgeometrie der 2. Grundform, Ber. Math.-Stat. Sekt. Forsch. Graz 219 (1984), 1-7.

[5] A. Martínez and F. Milán, Affine isoperimetric problems and surfaces with constant affine mean curvature, Manuscr. Math. 75 (1993), 35-41.

[6] K. Nomizu and T. Sasaki, Affine differential geometry, Cambridge University Press, 1994.

[7] H. Pabel and R. Keilbach, Die zentroaffine Familie von Relativnormalen und ihre Minimalflächen, 25. Süddeutsches Differentialgeometrie-Kolloquium (Wien, 2000), H. Stachel et al. (eds.), TU Wien, Institut für Geometrie, 2001, 9-15.

[8] U. Simon, Minkowskische Integralformeln und ihre Anwendungen in der Differentialgeometrie im Großen, Math. Ann. 173 (1967), 307-321.

[9] U. Simon, A. Schwenk-Schellschmidt, and H. Viesel, Introduction to the Affine Differential Geometry of Hypersurfaces, Lecture Notes, Science University of Tokyo, 1991.

[10] L. Verstraelen and L. Vrancken, Affine variation formulas and affine minimal surfaces, Michigan Math. J. 36 (1989), 77-93.

[11] C. P. Wang, Centroaffine minimal hypersurfaces in $\mathbb{R}^{n+1}$, Geom. Dedicata 51 (1994), 63-74.

[12] M. Wiehe, Deformations in affine hypersurface theory, Ph.D. thesis, Fachber. Math., TU Berlin, 1998, Shaker Verlag, Aachen, 1999. 